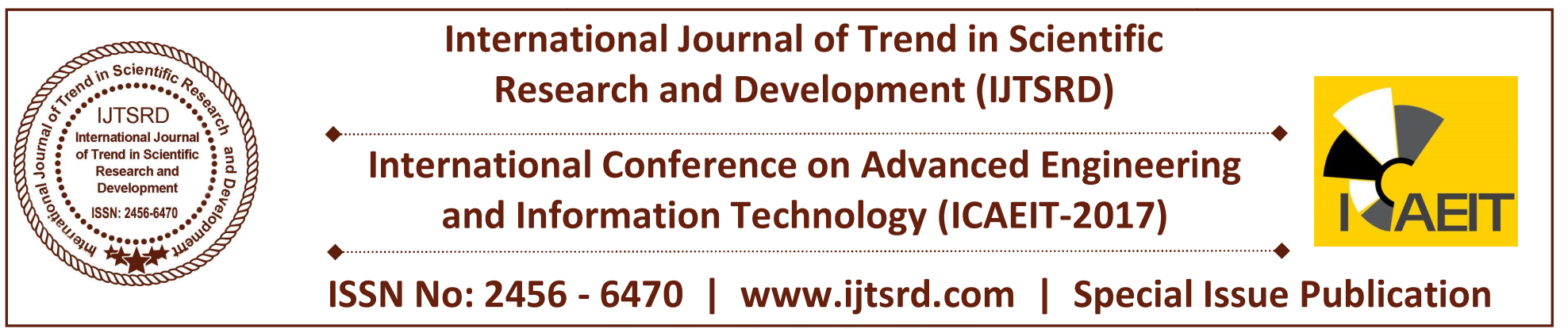

\title{
Automation of Medical Waste Separation using Advanced Technologies to Minimize its Impact on Environment
}

\author{
Ramani Bai V. G. ${ }^{1}$, Alla Kay R. ${ }^{2}$, Andy Chan $^{3}$ \\ ${ }^{1}$ Faculty of Built Environment, Linton University College, Mantin, Negeri Sembilan, West Malaysia \\ ${ }^{2}$ Faculty of Engineering and Technology, Linton University College, Mantin, Negeri Sembilan, West Malaysia \\ ${ }^{3}$ Faculty of Engineering, University of Nottingham Malaysia Campus, West Malaysia
}

\section{ABSTRACT}

This paper describes a shape recognition technique using boundary chain codes extracted by a method as described by Pavlidis and used an 8-connected neighbourhood. A chain code is a representation of a two-dimensional contour using a one-dimensional array. Feed forward neural networks were trained to recognise these chain codes. In addition, backpropagation network is trained using different training algorithms and the resulting optimal parameters are recorded. Depending upon the complexity of the object to be recognised, this technique can used to form the basis for object recognition or as the best method. The research is also aimed to compare the performance of chain code representation as against centroidal profile extraction. The third objective is to determine the effectiveness of Feed forward artificial neural networks - ANNs in recognising and classifying different medical waste items in the image form. The networks were trained on a large number of medical waste items. The wide variety of shapes and textures revealed that just a representation of an object's boundary is not sufficient to recognise every object in the set, and some form of texture recognition will also be required in recognising medical wastes. The results have shown that chain code has lesser performance as compared to centroidal profile representation.

Keywords: Occupational health, Medical waste, Chain codes, Feedforward Networks, Waste Classification, Hazardous wastes.

\section{INTRODUCTION}

With the increasing use of disposable equipment, medical waste is being generated at an increasingly rapid pace. While some countries do recycle medical waste, incineration is still the primary and occasionally the sole means of medical waste disposal in many countries, including Malaysia. A strong concern with medical waste recycling is its infectious nature, which makes sorting by humans a very hazardous activity. To automate this process, we seek to develop a system to ensure proper segregation of medical waste.

WHO guidelines suggests incineration be used as an interim solution and promotes non-burn technologies for medical waste disposal in the long term [1] and [12]. A research conducted by Yoan-Kagoma, the medical director has been concluded that the proper waste segregation is the single most effective cost saving measure. Therefore, he has suggested that all the hospital should compulsory adopt this into practice [11]. Addition to this, a hospital should reduce their ecological footprints by having comprehensive medical waste segregation and adopt "reduce, reuse, recycle" concept into medical waste management system [10]. Moreover, without designated medical waste segregation, enormous waste from medical institutional especially operation room is being classified as

'biohazard waste'. 
Many items that can be disinfected using chemical or physical methods (e.g. autoclaving) and then recycled are simply incinerated instead. Having official medical waste recycling programs may also play a role in curbing illicit medical waste scavenging and recycling activities that emerge due to lack of regulation [2] and [3].

Medical waste generated in Malaysia increased from 3203 tonnes in 1997 to 14,140 tonnes in 2008 , and is projected to rise to over 30,000 tonnes by 2015 [5]. The increasing use of disposable equipment plays a considerable role in this rise. Another concern is the amount of items mistakenly classified as infectious waste for incineration, thereby increasing the costs and risks involved (as some of these items may not be designed to be incinerated). A study carried out over eight years between 1991 and 1999 in Dhahran Health Centre, Saudi Arabia [6] discovered several misclassified items that could pose risks to workers sorting waste or to the incinerator itself.

Even after the implementation of a segregation program, a third of paper waste sent for incineration consisted of blue wraps used to cover sterilised equipment (although technically the blue wrap is made of polyethylene, a plastic). A study of the waste and recycling practices of five hospitals and three animal hospitals in Massachusetts, USA suggested a revision of medical waste regulations to increase recycling of plastics used in hospitals [7]. The Malaysian Department of Environment defines major classification of Clinical Wastes into 5 broad classes [8].

Many developed nations have medical waste legislation; however there is generally little guidance as to which objects can be defined as infectious. This lack of clarity has made sorting medical waste inefficient, thereby increasing the volume of waste treated for pathogens, which is commonly done by incineration. The review concludes that better education of healthcare workers and standardized sorting of medical waste streams are key avenues for efficient waste management at healthcare facilities, and that further research is required given the trend in increased medical waste production with increasing global GDP [13].

The medical waste generation rate, diagnosis-related groups score, case-mix index score, and adjusted generation rate were calculated. The comparisons were conducted by dividing included hospitals into groups according to level and affiliation. Although the effects on generation rate by medical activities in outpatient department need to be studied further, and the application is limited by the support of a full equipped hospital information system and a sound quality control of clinical data, it is still a simple and operable tool to compare the performance of hospital medical waste management [15]. The mismanagement of regulated waste is a costly issue for many health care facilities. Our facility undertook a quality improvement project to determine whether increasing staff members' knowledge of regulated-waste management would reduce its mismanagement in the surgical setting. Personnel achieved a $41 \%$ reduction in the total mass of regulated waste sampled and a $77 \%$ reduction in nonregulated item mass. The results of the audits determined that perioperative staff members are more likely to improve compliance with the disposal of regulated waste after increasing their knowledge base [16].

From an environmental standpoint, an increasing number of studies are assessing the environmental impacts of medical devices and the processes by which they are utilized. The aim of this study was to fill these knowledge gaps by using life cycle assessment (LCA) and life cycle cost assessment (LCCA) to model the environmental and economic impacts of medical device supply chains when varying levels of reprocessed devices are used at Phoenix Baptist Hospital (PBH) in Phoenix, Arizona. The LCA included all cradle-to-grave processes for the seven medical devices. Results of the study showed that if inputs (i.e., ethylene oxide, water, electricity) were optimized, the use of reprocessed devices offers global warming, human health, and economic benefits over the same devices used as disposables [17].

This study aims to perform the sharpening of the dental $x$-ray image in the form of a panoramic dental $\mathrm{x}$-ray. The method used in this study was segmentation morphology consisting of the dilation, erosion and gradient process. This study also developed a process of morphology gradient of subtracting morphology dilation results with the results of morphology erosion dilation in iterating basis. The results achieved indicate that the image enhancement process in each iteration stage can display caries objects clearly, making it easier to identify proximal caries. In this study have been 
compiled a looping morphology gradient algorithm which is called multiple morphology gradient [18]. This study also used mathematical morphology in detecting objects, by using dilation operation and filling holes. After the process of mathematical morphology was completed, the next process was motif extraction by applying Moore contour tracking algorithms and the development of chain code algorithms. The results of the process carried out showed that the development chain code algorithm can generate the number of objects, the length of chain code, and probable value of rate of appearances of each chain code in a motif, despite there are some objects in a motif. Then the values are stored into the database as The Feature of

Songket Motifs[19].

Colour appearance attributes in the CIELab colour model (L-Lightness, $\mathrm{a}$ and $\mathrm{b}$ are colour-opponent dimension) are used to generate colour appearance feature vectors namely the saturation metric, lightness metric and multicoloured metric. For the categorizations, we use the Nearest Neighbour (NN) method to detect the classes by using the predefined colour appearance descriptor measures and the pre-set thresholds. The experimental results show that the quantization of CIELab colour model into 11 uniformly bins for each component had achieved the optimum result for all colour appearances categories [20].

This paper presents an algorithm of using digital panoramic dental x-ray images to detect dental caries. Type of algorithm used in this study is normal $\mathrm{mMG}$, Enhancement mMG, and Smooth mMG. This study makes use of MATLAB and it performs dental caries detection in three algorithms. A dataset of 225 digital panoramic dental x-ray images in .png format is used to edge detection of the object in dental. The results are helpful to identify such caries from the tooth [21].

\subsection{Medical Waste Classification}

A simple classification system based on the nature of the hazard posed by the object can be seen in Error! Reference source not found.. This has been partially derived from the Malaysian Ministry of Environment guidelines. A more detailed list including subclasses can be seen in Table 2 .
Table 1: Proposed Classification and codes

\begin{tabular}{|c|c|}
\hline Class & Code \\
\hline Microbiological Waste & N261A \\
\hline Pathological Waste & N261B \\
\hline Sharps & N261C \\
\hline Pharmaceutical Waste & N251D \\
\hline Radioactive Waste & N251E \\
\hline Chemical Waste & N251F \\
\hline Other Waste (unclassified) & O \\
\hline
\end{tabular}

\section{Data collection and Pre-Processing}

The networks were trained with photographs of individual medical waste items. The objects are assumed to be on a conveyor belt in a sorting facility, hence lighting is constant and angle of rotation is to be locked on a single plane facing the camera. Photographs of medical waste were taken with the camera perpendicular to the plane of the object, on a plain white or black background. A breakdown of the number of photographs per class and subclass is given in. The overall stages of development of a model for AUTOM (named, 'AUTOmated Medical Waste Segregation) is shown in Figure 1.

Several pre-processing operations are performed before a clean outline of the object may be extracted. These are listed in order:

1. Resizing the image

2. Background removal

3. Conversion to a binary format

4. Salt and pepper noise removal

5. Edge detection

6. Removal of small objects

This results in a clean image suitable for boundary extraction.

Table 2: Number of training images

\begin{tabular}{|c|c|}
\hline Class & Code \\
\hline Microbiological Waste & N261A \\
\hline Pipette tips & 2 \\
\hline Slides & 4 \\
\hline Vaccine bottles & 14 \\
\hline Pathological Waste & N261B \\
\hline Sharps & N261C \\
\hline Syringes & 10 \\
\hline Scalpel blades & 10 \\
\hline Broke glass equipment & 7 \\
\hline Slides & 4 \\
\hline Pharmaceutical Waste & N251D \\
\hline Radioactive Waste & N251E \\
\hline Chemical Waste & N251F \\
\hline Other Waste (unclassified) & O \\
\hline
\end{tabular}




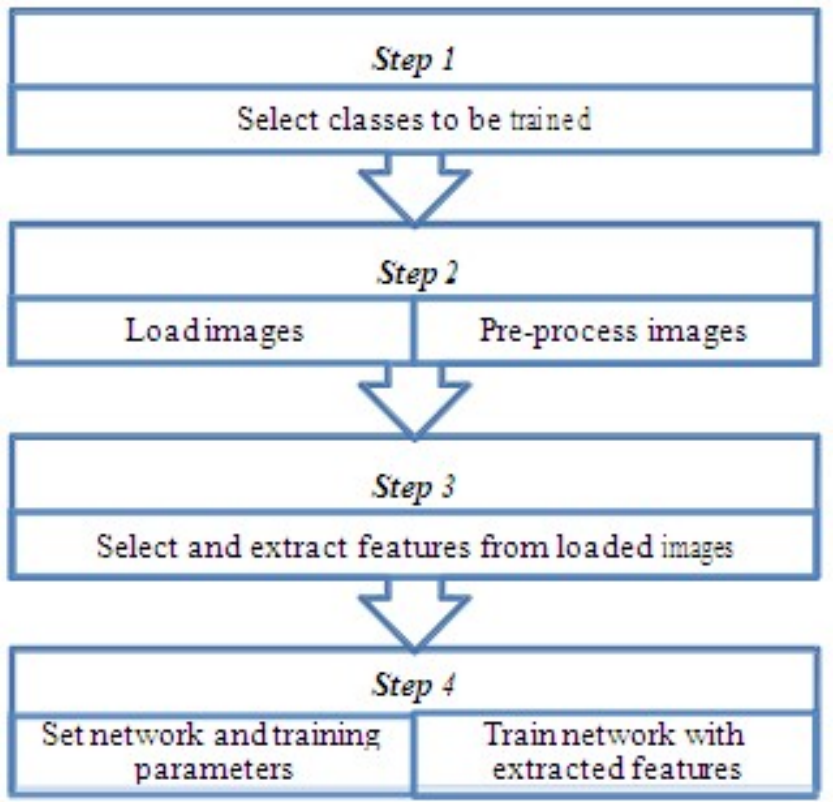

Figure1. Generic Stages in AUTOM Development

\section{Development of AUTOM}

The application of artificial neural networks and image processing to develop the AUTOM model for solving the problem of medical waste segregation by Machines rather than human is described in following sections (Figure 2).

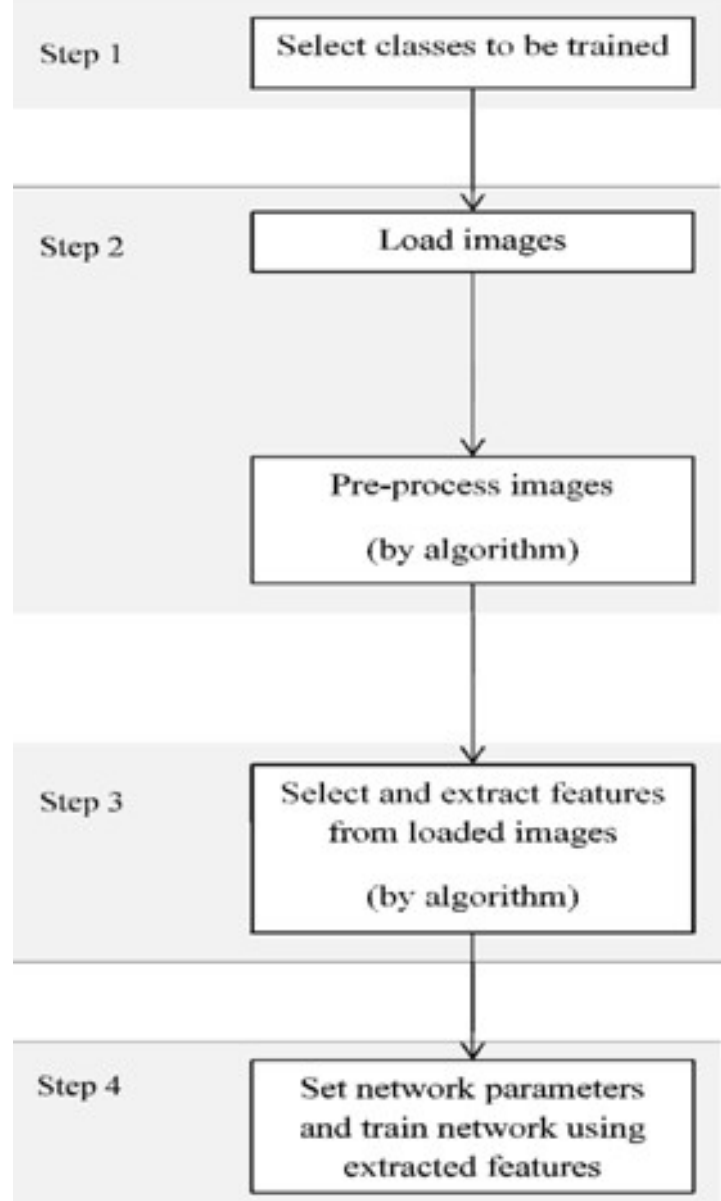

Figure2. Algorithm of development of AUTOM (Automated Medical Waste Segregation) machine

\subsection{Detailed Algorithm of AUTOM}

The details on the algorithm of AUTOM development, training, testing and validation area also presented in the following section and are shown in Figure. 3 and Figure4.

\subsubsection{Pre-processing Step:}

1. Resize to $640 * 480$ pixels (imresize)

2. Convert to grayscale (rgb2gray)

3. Adjust grayscale intensities (imadjust)

4. Convert to binary using Otsu's method for thresholding (im2bw, graythresh)

5. Fill holes (imfill)

6. Create two structuring elements, 7 pixels long, one horizontal and one vertical (strel)

7. Dilate and erode image using two structuring elements (imdilate, imerode)

8. Remove objects with an area of less than 50 pixels.(bwareaopen)

\subsubsection{Feature extraction Steps:}

1. Detect objects in the binary image (bwconncomps)

2. Trace boundaries of each object (regionprops, 'PixelList')

3. Extract list of boundary pixels for each object (bwtraceboundary)

4. Derive desired shape feature from the lists:

a. Chain code: encode direction from one pixel to the next (Euclidean distance formula)

b. Centroidal profile: calculate distance of each pixel from the object's centroid (regionprops, 'Centroid').

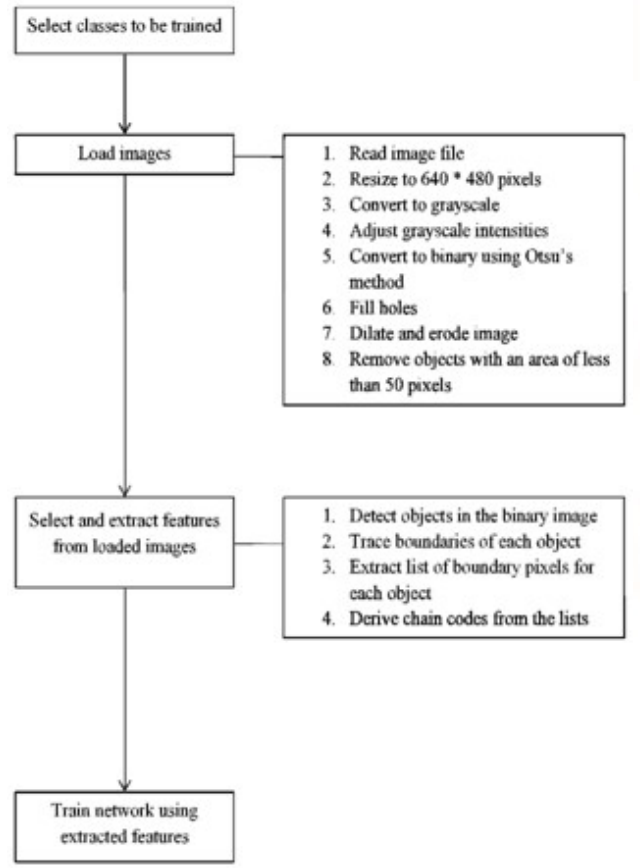

Figure3. AUTOM Program Flowchart: Training 


\subsubsection{Training Steps:}

1. Create array of features (network input)

2. Create array of classification vectors based on earlier choices (network output)

3. Create a new pattern net with a hidden layer with 40 neurons

4. Disable network input and outputs processFcns

5. Increase max_fail to 12 (number of successful validation checks)

6. Train the network using the Levenberg- Marquadt algorithm

7. Save the trained network.

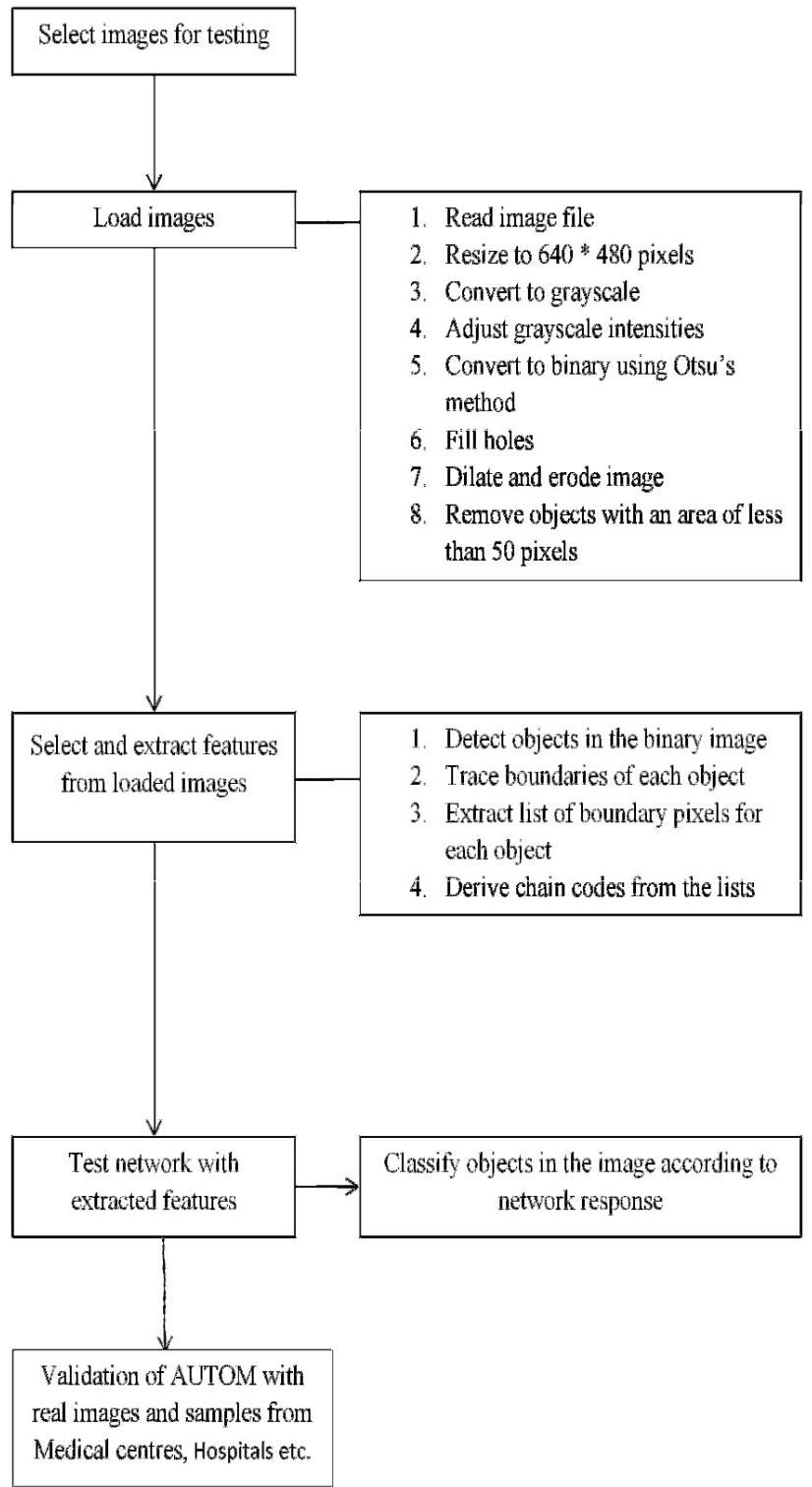

Figure4. Program Flowchart: Testing and Validation

\subsubsection{Testing:}

1. Load the network and classification file

2. Load and pre-process images and extract features

3. Simulate the network with the extracted features

4. Classify images based on network

5. Proceed for validation with real images.

6. Chain Code Representation
A contour in a typical digital image can be described as list of $x$ - and $y$-coordinates, each leading to the next. This usually results in two arrays, and while this is not a problem for most software, an input to a neural network must be presented as a single array. There are several methods of extracting and representing an edge contour in this manner, however, due to their discrete nature, axis crossover, centroidal profile and chain code representations are more tolerant to noise and small variations in shape. For this paper, chain codes are tested, and other representations will be tested in the future.

A chain code follows a contour in a clockwise or anticlockwise direction and an element in the code array points to the next pixel in the contour. A chain of coordinates is now represented by a chain of directions. Another advantage to this representation is the possibility of scale and limited rotation invariance. For this study, the chain code is extracted using the method described as by Pavlidis [9], and used an 8connected neighbourhood.

\subsection{Differential Chain Code}

A differential chain code is a small but important variation of the chain code, in which only changes in the direction (rather than the absolute direction) of the chain are assigned values, while straight lines are represented by zeroes. This can be derived by finding the "differential" of the chain code (the 8 modulus of the difference between two consecutive elements). In MATLAB, this can be implemented by mod (diff(x), $n$ ) where $x$ is the chain code array and $n$ is number of neighbours (4 or 8$)$.

\subsection{Normalisation}

The length of the chain code corresponds to the length of the perimeter of the object in the image (in pixels), which doesn't necessarily reflect on its actual size. Size invariance was decided on for this project and hence the length of all extracted chain code arrays are normalised to 100 elements using MATLAB's built in interp1 function.

\subsection{Neural Network Training}

Feed forward neural networks were trained using the extracted features with a variety of backpropagation algorithms.

The default configuration is shown in Table 3. The network performance was also tested while varying a single parameter while keeping the others at the default value. 
Table 3: Default training and pre-processing parameters

\begin{tabular}{|c|c|}
\hline Parameter & Value \\
\hline Hidden layers & 1 \\
\hline Neurons & {$[40]$ neurons } \\
\hline Training algorithm & Levenberg-Marquadt \\
\hline Image size & $200 * 200$ \\
\hline Chain size & 100 elements \\
\hline Noisy samples & 100 \\
\hline
\end{tabular}

\subsection{Network output}

The network output is a two element variable, each element ranging from 0 to 100: the closer to 100 the output is, the higher the likelihood of the object belonging in that class. The first element corresponds to the object belonging to the class, while the second element represents all other classes.

\subsection{Training set}

The training set for a particular class was trained against images from other classes to strengthen the classification performance of the network.

\subsection{System Specifications}

All operations were performed on a 64-bit installation of MATLAB 2012a running on Windows 7. The software runs on a machine with an Intel Core i7 $2860 \mathrm{QM}$ processor running at $2.50 \mathrm{GHz}$ and has $32 \mathrm{~GB}$ of DDR3 RAM.

\section{Results and Discussion}

The paper has described the development of the ANN model for medical waste recognition and classification based on image processing. Figures 1 through 4 explain the detailed development procedure for medical waste segregation using ANN and Image processing. The current research has compared two methods of image processing under shape recognition namely, Chain code and Centroidal profile methods. Based on the results it was found that Centroidal profile perforamed better as compared to Chain code. The results are shown in Figure 5 and Figure 6 . Almost $39 / 40$ objects were recognised to $100 \%$ by centroidal profile as compared to $36 / 40$ by Chain code representation.

The output of a network trained with $\mathrm{x}$ classes is a single vector with $\mathrm{x}$ elements, each class represented by a single element. The value of an element ranges from 0 to 1 . Higher the value of an element, higher is the likelihood of the input belonging to the class. The class corresponding to the element with the highest value is assigned to the input. Ideally, the value of the corresponding element is 1 while the others are 0 . These ideal outputs are used to train the network.

Experimentally, the output was not so clean in the beginning.

The trained feed forward network with the following parameters has shown good performance in recognising and classifying different medical waste items from images and videos. The effective ANN has 40 hidden neurons, time taken 78 seconds (varies widely, highest: 8 hours), training set size is 141 images +141 x 25 noisy samples $=3666$ samples the algorithm used is Levenberg-Marquadt. The time taken for running was increased with a larger training set.

Medical waste poses a serious public health problem as it contains highly toxic chemicals, pathogenic viruses and bacteria. A proper classification of medical waste as developed through this study would improve hygiene, reduce improper disposal, and reduce the spread of disease from a medical setting to the general public. The medical centres can ensure for occupation health and safety in their organization. Moreover the current medical classes are $90-95 \%$ over classified.

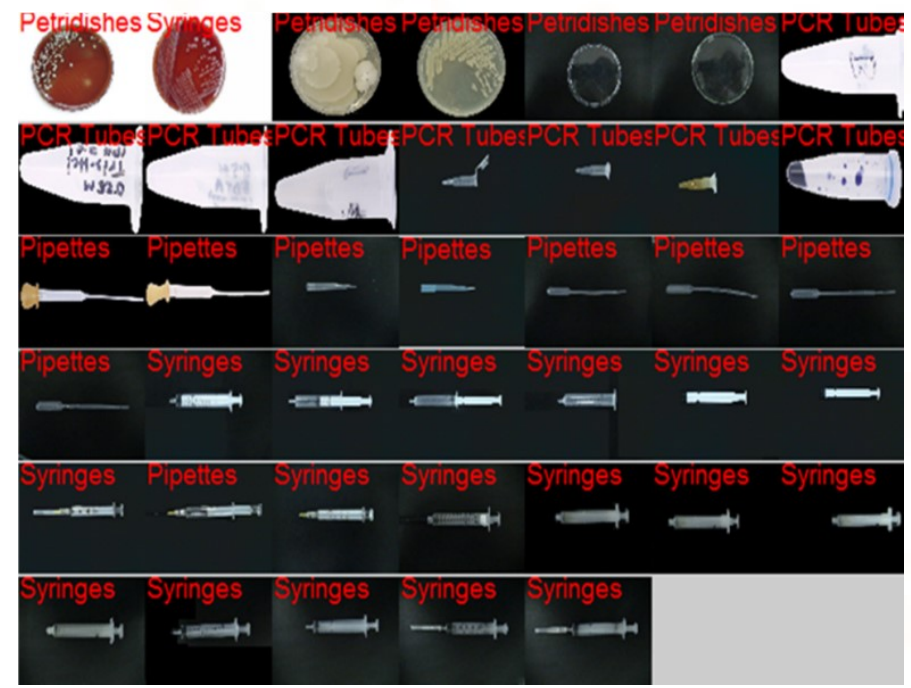

Figure5. Shape recognition results using Chain code 


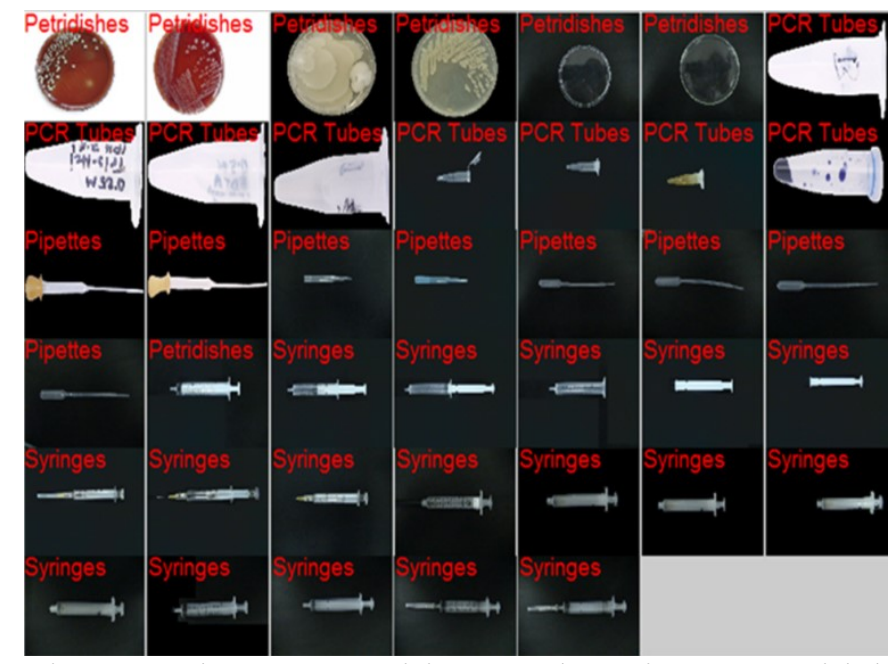

Figure6. Shape recognition results using Centroidal Profile method

\section{Conclusion}

In the current study, by merging Malaysia's Scheduled Waste Regulations and European Waste Codes the new classification serves as a promising one for the future. The burden of infectious waste in every facility and ecological footprints will be highly reduced. The process of development of a model for recognition and classification of the newly developed medical classes has been successfully presented in this paper. The performaces of two different image processing methods have been studied. Shape Recognition by Centroidal Profile in which each pixel in the traced boundary is represented by its distance from the object's centroid and has performed better than the outline encoded method (chain code). The testing confirmed that ANNs are capable of recognising rigid objects by their shape alone. ANNs require a one dimensional input. The contour of the object must be represented by a 1-D array. Feedforwad networks trained by LevenbergMarguadt proved to be the better performing network in this study.

Thus the developed model can be further being studies and developed into an instrument for proper segregation of medical wastes. When deployed to an automated waste segregation instrument (named, 'AUTOmated Medical Waste Segregation- AUTOM') in laboratories and medical centres for segregating the medical wastes, it will avoid human intervention to segregate the waste.

\section{Acknowledgement}

The authors would like to acknowledge and thank MOSTI (Ministry of Science and Technology),
Malaysia for their funding for this project and that has resulted in the publication.

\section{References}

1. World Health Organization (WHO), "Health-care waste management," vol. Fact sheet $\mathrm{N}^{\circ} 281$, October 2011.

2. Patwary M. A., O'Hare W. T., Sarker M., "An illicit economy: Scavenging and recycling of medical waste", Journal of Environmental Management, 92(11), 2900-2906, 2011.

3. J. I. Blenkharn, "Medical wastes management in the south of Brazil", Waste Management, 26(2006), 315-317, 2006.

4. R. R. A. M Mato and M. E. Kaseva, "Critical review of industrial and medicalwaste practices in Dar es Salaam City," Resources, Conservation and Recycling, 25, 3-4, 1999.

5. Pawel Suwinski and Nurul Fatiha, "Opportunity and money lies in Malaysia's hospital waste management market", Frost \& Sullivan, 2010.

6. David L. Hagen, Faisal Al-Humaidi, Martin A. Blake, "Infectious waste surveys in a Saudi Arabian hospital: An important quality improvement tool," American Journal of Infection Control, 29(3), 198-202, 2001.

7. Byeong-Kyu Lee, Michael J. Ellenbeckerb, Rafael Moure-Erasob, "Analyses of the recycling potential of medical plastic wastes," Waste Management, 22(5), 461-470, 2002.

8. Deparment of Environment, Ministry of Natural Resources and Environment, Malaysia, "Guidelines on the Handling and Management of Clinical Wastes in Malaysia," Third Edition, 2009.

9. Theodosios Pavlidis, "Algorithms for graphics and image processing”, Computer Science Press, 1982.

10. Kralj D., Markic M., "Building Materials Reuse and Recycle", WSEAS Transactions on Environment and Development, 4, 409-418, 2008.

11. Kagoma, Y., Stall, N., Rubinstein, E., Naudie, D., "People, planet and profits: the case for greening operating rooms", Can. Med. Assoc. J., 184(17): 1905-1911, 2012.

12. World Health Organisation, "Safe health-care management", Policy Paper, World Health Organization, 2004. 
International Journal of Trend in Scientific Research and Development (IJTSRD) | ISSN: 2456-647

13. Elliott Steen Windfeld, Marianne Su-Ling Brooks, Medical waste management - A review. Journal of Environmental Management, 163, 98-108, 2015 .

14. World Health Organization (WHO), "Health-care waste management," Fact sheet N²81, October 2011.

15. Yantao Xin, "Comparison of hospital medical waste generation rate based on diagnosis-related groups", Journal of Cleaner Production, 100, 202207, 2015.

16. Kristen Perrego, "Improving Staff Knowledge of Perioperative Regulated-Waste Management", AORN Journal, 105(1), 85-91, 2017.

17. Scott Unger, Amy Landis, 2016. Assessing the environmental, human health, and economic impacts of reprocessed medical devices in a Phoenix hospital's supply chain. Journal of Cleaner Production, 112(3), 1995-2003.

18. Jufriadif Na`am, Johan Harlan, Sarifuddin Madenda, Eri Prasetio Wibowo, "Identification of the Proximal Caries of Dental X-Ray Image with Multiple Morphology Gradient Method",
International Journal on Advanced Science, Engineering and Information Technology, 6 (2016): 3, 345-348, 2016.

19. Yuhandri, Sarifuddin Madenda, Eri Prasetyo Wibowo, Karmilasari, "Object Feature Extraction of Songket Image Using Chain Code Algorithm”, International Journal on Advanced Science, Engineering and Information Technology, 7(2017):1, 235-241, 2017.

20. Aniza Othman, Tengku Siti Meriam Tengku Wook, Shereena M. Arif, "Quantization Selection of Colour Histogram Bins to Categorize the Colour Appearance of Landscape Paintings for Image Retrieval", International Journal on Advanced Science, Engineering and Information Technology, 6 (2016), 930-936, 2016.

21. Jufriadif Na'am, Johan Harlan, Sarifuddin Madenda, Eri Prasetio Wibowo, "The Algorithm of Image Edge Detection on Panoramic Dental XRay using Multiple Morphological Gradient (mMG) Method" International Journal on Advanced Science, Engineering and Information Technology, 6 (2016), 1012-1018, 2016. 